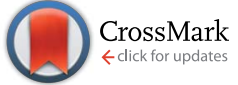

Cite this: J. Mater. Chem. A, 2014, 2 , 16669

Received 10th May 2014

Accepted 12th August 2014

DOI: $10.1039 / c 4 t a 02363 c$

www.rsc.org/MaterialsA

\section{Electrospun chitosan-polyvinyl alcohol composite nanofibers loaded with cerium for efficient removal of arsenic from contaminated water}

\author{
Reena Sharma, ${ }^{d}$ Nahar Singh, ${ }^{b}$ Ashish Gupta, ${ }^{a}$ Sangeeta Tiwari, ${ }^{d}$ \\ Sandeep Kumar Tiwari ${ }^{c}$ and Sanjay R. Dhakate*a
}

Contamination of water due to arsenic has been extensively reported all over the world. It has led to massive epidemics of arsenic poisoning. An urgent need is being felt to develop efficient techniques for the removal of arsenic from contaminated water. In this context, cerium (Ce) loaded chitosan (CHT)-polyvinyl alcohol (PVA) composite (Ce-CHT/PVA) nanofibers were developed by electrospinning technique and have been employed for removing As(III). The Ce-CHT/PVA composite nanofibers efficiently adsorb As(III) and purify water below the prescribed limit of WHO/EPA. As(III) adsorption over the surface of Ce-CHT/PVA has been confirmed by scanning electron microscopy and energy dispersive $\mathrm{X}$-ray spectroscopy (SEMEDAX), Fourier transform infrared spectroscopy (FTIR) and X-ray diffraction (XRD). The quantitative estimation of As(III) has been carried out by flameless atomic adsorption spectrophotometer-hydride generator (AAS-HG) system. The As(II) adsorption efficiency of Ce-CHT/PVA composite nanofibers has been established as a function of $\mathrm{pH}$, time, temperature and adsorbent dose. The adsorption data were best fitted to Langmuir isotherm, and the maximum adsorption capacity $\left(q_{\mathrm{m}}\right)$ was found to be $18.0 \mathrm{mg}$ $\mathrm{g}^{-1}$. The interference studies of several ionic species individually as well multi-element for As removal have also reported. The measurement of the uncertainty of As(III) determination was calculated after determining the contributing factors. The data are reported with a confidence level of $95 \%(K=2)$. The $\mathrm{Ce}-\mathrm{CHT} / \mathrm{PVA}$ composite nanofibers are non toxic and can be directly used for water purification or after being embedded in the form of membrane or candles.

\section{Introduction}

The natural as well as anthropogenic contamination of the environment with metallic elements, as defined by the United State-Environmental Protection Agency (US-EPA), are of major concern because of their toxicity, bio-accumulating tendency, and threat to human life and environment. ${ }^{1-3}$ The most common metallic elements for human poisoning are mercury, cadmium, lead, arsenic, chromium, copper and zinc. Some of them are carcinogenic. Arsenic (As), a metalloid, is highly toxic and causes neurologic, cardiovascular, gastrointestinal and hepatic, and dermal disorders in human beings. Several diseases in human being, such as 'Kai Dam' in Thailand, 'Bell Ville' in Argentina,

${ }^{a}$ Physics \& Engineering of Carbon, Division of Material Physics \& Engineering, CSIR-National, Physical Laboratory, New Delhi-110012, India. E-mail: dhakate@ mail.nplindia.org; Fax: +91 11 45609310; Tel: +91 1145608257

${ }^{b}$ Analytical Chemistry Division, CSIR-National Physical Laboratory, New Delhi110012, India

${ }^{c}$ Council of Scientific and Industrial Research, Rafi Marg, New Delhi 110001, India ${ }^{d}$ Amity Institute of Applied Sciences, Amity University, Noida, 201303, UP, India

$\dagger$ Electronic supplementary information (ESI) available. See DOI: $10.1039 / \mathrm{c} 4 \mathrm{ta} 02363 \mathrm{c}$
'Black Foot' in Taiwan, tummy bug, cancers of lungs and skin, are caused by the consumption of As contaminated water. ${ }^{4}$ As can exist in natural water both in inorganic and organic forms. In natural water, it exists in two oxidation states, arsenite (As III) and arsenate (As v). The inorganic As is 70 times more toxic than organic species, and within this class, As(III) is ten times more toxic than $\operatorname{As}(\mathrm{v}){ }^{5,6}$ It enters in the environment and water through geochemical reactions, industrial waste discharge, etc. The long-term consumption of As contaminated water has harmful impacts on human beings. According to the European Commission and US-EPA, the permissible maximum limit of As in drinking water is $10 \mu \mathrm{g} \mathrm{\textrm {L } ^ { - 1 }}$. $^{7}$

To overcome the problem of As in drinking water, various technologies, such as oxidation, filtration, coagulation, ion exchange, adsorption and membrane separation, ${ }^{8,9}$ have been employed. It has been observed that the removal of As(III) is more tedious than $\mathrm{As}(\mathrm{v})$ by filtration. ${ }^{\mathbf{1 0}}$ Adsorption processes are commonly developed due to their simplicity and ease of handling. The common adsorbents for arsenic removal include iron oxides, ${ }^{11,12}$ iron-impregnated chitosan, ${ }^{13}$ aluminum compounds, sand, activated carbon, ${ }^{14}$ silica ceramics, ${ }^{15} \mathrm{Ce}-\mathrm{TiO}_{2}$ adsorbent, ${ }^{16}$ urchin-like $\alpha$-FeOOH hollow spheres ${ }^{17}$ and nanostructure-material-like graphene based hybrid for electrocatalyst. ${ }^{18}$ In recent 
times, biopolymers-like chitin and chitosan (CHT) are being considered as suitable low cost adsorbents for the removal of heavy metals due to their biocompatibility, biodegradability and hydrophilicity. ${ }^{19}$ Chitosan has emerged as the biomaterial of choice for several applications based on its film-forming capabilities, metal-binding capacity, biodegradability, antimicrobial activity and wound healing capacity. ${ }^{20}$ The positively charged amino group over $\mathrm{CHT}$ at $\mathrm{pH} 6$ reacts with negatively charged metal ions and microbes. ${ }^{21-23}$ The CHT can be transformed into chitosan beads, ${ }^{24}$ iron-impregnated chitosan, porous chitosan/ $\mathrm{Fe}_{3} \mathrm{O}_{4} / \mathrm{Fe}(\mathrm{OH})_{3}$ microsphere, ${ }^{25}$ iron-chitosan composite granules, ${ }^{26}$ alpha- $\mathrm{Fe}_{2} \mathrm{O}_{3}$ impregnated chitosan beads, ${ }^{27}$ chitosan-Fecross linked complex ${ }^{28}$ and Ce-loaded cation exchange resins ${ }^{29}$ for the purification of water, especially from As(III). Because the As(III) adsorption capacity of CHT is quite low, it requires longer exposure for effective adsorption in comparison to other adsorbents reported in literature.

Therefore, Ce(III) is loaded in the composite nanofiber to improve the adsorption capacity and reduce the adsorption time of CHT. It is observed that because of smaller ionic radii, high electric charge and higher potential energy associated with $\mathrm{Ce}$, the adsorption behavior of Ce-CHT/PVA composite nanofibers improves significantly while the optimal exposure time is also drastically reduced. On the addition of Ce(III) in CHT/PVA, the stability and attracting capability of composite nanofibers toward anionic species increases due to increasing positive charge on the surface of composite nanofiber. ${ }^{30}$ However, because of crystal formation and H-bonding in D-glucosamine unit of CHT, it is difficult to electrospun fibers from the highly viscous solution. ${ }^{31,32}$ To reduce the viscosity of chitosan, polyvinyl alcohol (PVA) is added. The PVA reduced intermolecular interactions between the chitosan molecules and facilitated electrospinning. Furthermore, PVA is water soluble, non-toxic, and has good biological activities in combination with chitosan. ${ }^{33}$ The present work reports the preparation of Ce-CHT/PVA composite nanofibers by electrospinning and investigates the adsorption and removal of As(III) from water.

\section{Experimental and characterization}

\subsection{Materials}

Analytical reagents grade chemicals were used: chitosan (M.W; 100 000-300 000) from Across Organic; polyvinyl-alcohol (M.W; approx. 125 000) from $\mathrm{CDH}$; formic acid and cerium(III) nitrate hexahydrate (M.W; 434.23) from Chemica-biochemica reagents; sodium borohydride $\left(\mathrm{NaBH}_{4}\right)$, hydrochloric acid $(\mathrm{HCl})$, sodium hydroxide $(\mathrm{NaOH})$, potassium nitrate $\left(\mathrm{KNO}_{3}\right)$ and potassium hydroxide (KOH) from E. Merck (India). Double distilled water was used for synthesis while de-ionized water of $18.2 \mathrm{~m} \Omega$ resistivity (Millipore, USA) was employed for analysis on atomic adsorption spectrophotometer-hydride generator (AAS-HG).

\subsection{Synthesis of Ce-CHT/PVA composite nanofibers by electrospinning}

The Ce-CHT/PVA composite nanofibers were prepared by the electro spinning equipment (ESPIN-NANO, procured from
Physics Instrument Company, Chennai) at optimized processing parameters discussed in earlier studies. ${ }^{34-36}$ For the preparation of Ce-CHT/PVA composite nanofibers, $4 \mathrm{wt} \%$ of CHT was dissolved in $2 \%(\mathrm{v} / \mathrm{v})$ formic acid under continuous stirring for $24 \mathrm{~h}$ at room temperature using a magnetic stirrer. $8 \%$ solution of PVA in acetic acid $(2 \%, \mathrm{v} / \mathrm{v})$ was added to the clear solution of CHT in a ratio of $7: 3$ (PVA : CHT) under continuous stirring for 4-5 h. In CHT/PVA solution, cerium(III) nitrate hexa-hydrate (1-6; w/w \%) was added with continuous stirring for $4-5 \mathrm{~h}$. The resultant Ce-CHT/PVA solution was filled in $2 \mathrm{~mL}$ syringe, electrospun at applied voltage of $20 \mathrm{kV}$ and $0.2 \mathrm{~mL} \mathrm{~h}^{-1}$ flow rate. The distance between the syringe tip and collector was maintained at $20 \mathrm{~cm}$. The composite nanofibers were collected on an aluminum foil wrapped on the collector. The Ce-CHT/PVA composite nanofiber prepared by this route exhibits hydrophilicity and some nanofibers disintegrate in water. To overcome this problem, the composite nanofibers were heated in an oven at $85{ }^{\circ} \mathrm{C}$ for 120 minutes.

\subsection{Characterization}

The morphology of Ce-CHT/PVA composite nanofibers before and after As(III) adsorption was studied by scanning electron microscope (SEM Model EVO M-1 of Zeiss), which was equipped with energy dispersive X-ray spectroscopy (EDAX) for the measurement of elemental composition. Functional groups present on the surface of composite nanofibers were investigated by FTIR spectrophotometer (Nicolet make, Nexus-47). The $\mathrm{X}$-ray diffraction patterns of the composite nanofibers before and after adsorption were recorded on X-ray Diffractometer (Model-Expert D6, Japan) at a wavelength of $1.5404 \AA$ and a $2 \theta$ angle between 5 and $80^{\circ}$. The concentration of As(III) in solution after adsorption with Ce-CHT/PVA composite nanofibers was analyzed using AAS-HG, (Model Vario-6-Analytik Jena, Germany) at a wavelength of $193.7 \mathrm{~nm}$. AAS-HG was calibrated using NIST (USA) SRM-3103a. For all the As(III) adsorption studies on composite, CSIR-NPL standard references were used (CSIR-NPL is the custodian of measurement standards in India). The VHG (UK) Multielement SM-75-100 standard reference material was used to check the mixed inference of various ions. All the reference solutions were used after subsequent dilutions. The individual solutions of various ionic species used in interference studies were prepared from high purity metals/ salts. A UV-vis spectrophotometer (Hitachi, Model-U3900H) was used to further establish the efficacy of the removal of As(III) from water.

\subsection{Determination of point zero charge $\left(\mathrm{pH}_{\mathrm{PZC}}\right)$}

The $\mathrm{pH}_{\mathrm{PZC}}$ of Ce-CHT/PVA was determined using potentiometric titration ${ }^{37}$ in nitrogen medium. $0.025 \mathrm{~g}$ of composite nanofibers were mixed in $25 \mathrm{~mL}$ of $0.03 \mathrm{M} \mathrm{KNO}_{3}$ solution and the system was kept overnight $(24 \mathrm{~h})$ under constant stirring to stabilize the $\mathrm{pH}$. Subsequently, $0.1 \mathrm{~mL}$ of $1 \mathrm{M} \mathrm{KOH}$ was added to the stabilized system. The $\mathrm{pH}$ was recorded after each addition of $0.05 \mathrm{~mL}$ solution of $0.1 \mathrm{M} \mathrm{HNO}_{3}$ using a micropipette. Blank titration $\left(0.03 \mathrm{M} \mathrm{KNO}_{3}\right)$ was also carried out in similar manner. 
The $\mathrm{pH}_{\mathrm{PZC}}$ was determined from the curve of $\mathrm{pH}$ against acid consumed (Fig. 1(a)).

\subsection{Adsorption experiments}

The adsorption experiments for the removal of As(III) were carried out by suspending composite nanofiber with a desired concentration of As(III) solution. The stock solution of As(III) was diluted in the desired range and $\mathrm{pH}$ was adjusted with of 0.001 $\mathrm{M} \mathrm{HCl}$ and $\mathrm{NaOH}$ solutions. The solutions along with Ce-CHT/ PVA composite nanofibers were stirred on a magnetic stirrer at a very low speed. The composite nanofibers can be easily separated from the solution without any filtration device. Finally, each sample was analyzed for remaining As(III) concentration by AAS-HG using 3\% sodium borohydride, $1.5 \% \mathrm{NaOH}$ and $2 \%$ $\mathrm{HCl}$ for arsine generation. Arsine atomizes at $900{ }^{\circ} \mathrm{C}$ using electro-thermal heating in the presence of argon gas with $36 \mathrm{~mL}$ $\min ^{-1}$ flow rate. ${ }^{38}$ The adsorption capacity $\left(q_{\mathrm{e}}\right)$ of Ce-CHT/PVA composite nanofibers for removing As(III) was calculated by eqn (1), while \% removal of As(III) by composite nanofibers was calculated by eqn (2).

$$
\begin{aligned}
\operatorname{Adsorption} \text { capacity }\left(q_{\mathrm{e}}\right) & =\frac{\left(C_{\mathrm{i}}-C_{\mathrm{f}}\right) \times V}{m} \\
\% \text { Removal of } \operatorname{arsenic}(\mathrm{III}) & =\frac{\left(C_{\mathrm{i}}-C_{\mathrm{f}}\right) \times 100}{C_{\mathrm{i}}}
\end{aligned}
$$

where, ' $C_{\mathrm{i}}$ ' and ' $C_{\mathrm{f}}$ ' was the initial and final concentration of $\operatorname{As}\left(\right.$ III) solution ( $\left.\mathrm{mg} \mathrm{L}^{-1}\right), V$; volume (L) used and $m$ is the weight of the composite nanofibers $(\mathrm{g})$.

The effect of $\mathrm{pH}$ on the adsorption of As(III) by Ce-CHT/PVA has been studied and illustrated in Fig. 1(b). It can be observed that As(III) is mainly adsorbed in the $\mathrm{pH}$ range of 6.2-7.0. Any increase or decrease in the $\mathrm{pH}$ of the test solution results in the decrease of the adsorption capacity of Ce-CHT/PVA. Deformation of nanofibers at lower $\mathrm{pH}$ values could be one of the reasons for the decrease in adsorption at lower $\mathrm{pH}$ values in addition to the $\mathrm{pH}_{\mathrm{pzc}}$ of the composite.

The adsorbent dose of Ce-CHT/PVA composite nanofibers is varied (2-20 mg/50 mL), while keeping fixed concentration of As(III) $3.5 \mathrm{mg} \mathrm{L}^{-1}$ in solution. $2 \mathrm{mg}$ of Ce-CHT/PVA composite nanofibers was stirred in $50 \mathrm{~mL}$ solution with a fixed quantity of As(III). Fig. 2 illustrates the effect of adsorbent dose on the

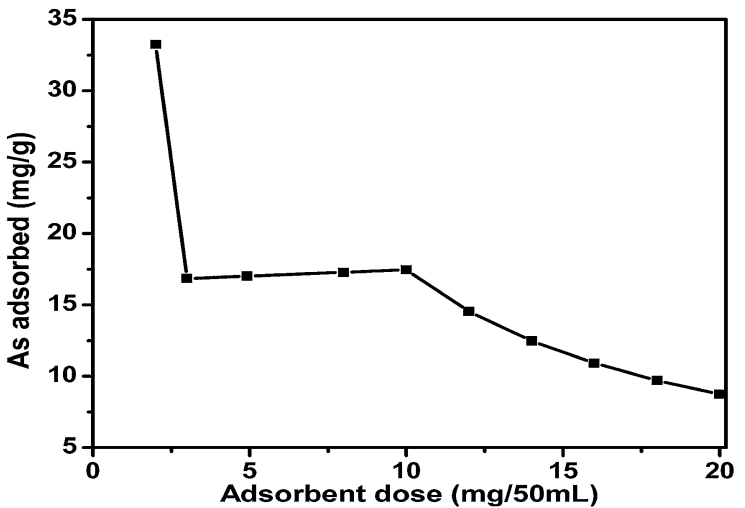

Fig. 2 Effect of Adsorbent dose on As(III).

adsorption of As(III). The maximum adsorption is observed for $2 \mathrm{mg}$ of nanofibers, which gradually decreases on increasing the weight of Ce-CHT/PVA composite nanofibers. The maximum adsorption observed for $2 \mathrm{mg}$ composite sample may be attributed to easy accessibility and free active sites on the surface of material. On increasing weight of Ce-CHT/PVA, the number of active sites increases but the ratio of As(III) to active sites decreases, resulting in a gradual decrease in adsorption. The optimum dose for As(III) is observed to be about $10 \mathrm{mg} / 50$ $\mathrm{mL}$ for $3.5 \mathrm{mg} \mathrm{L}^{-1}$ of As(III) solution.

\section{Results and discussion}

\subsection{Morphology of composites nanofibers by SEM and EDAX}

Chitosan is a linear cationic biopolymer soluble in most of the acids, and the protonation of chitosan changes it into a polyelectrolyte, which increases its viscosity. The repulsive forces between ionic groups within polymer backbone, arising due to the application of high electric field during electrospinning, restrict the formation of continuous nanofibers and often produce beads, ${ }^{39}$ which makes it difficult to fabricate pure CHT nanofibers. Therefore, PVA polymer is added to control the intermolecular interactions between the CHT molecules and the viscosity of the solution. After optimizing various electrospinning parameters, bead free nanofibers were prepared by employing PVA with chitosan in a ratio of $7: 3$. After CHT/PVA


Fig. 1 (a) Potentiometric curve for $\mathrm{pH}_{\mathrm{PzC}}$ determination and (b) effect of $\mathrm{pH}$ on $\mathrm{As}(\mathrm{III})$ adsorption. 
polymer fabrication, varying weight fraction of cerium(III) nitrate hexahydrate $(0.5 \%$ to $5 \mathrm{wt} \%)$ were added to achieve Ce rich nanofibers. It is observed that at $3.5 \mathrm{wt} \%$, cerium(III) nitrate hexahydrate in CHT/PVA solution gives bead free continuous composite nanofibers. To confirm the adsorption of As(III) on the Ce-CHT/PVA nanofiber, the samples were analyzed for elemental distribution by EDAX and elemental mapping (ESI, Fig. S1 $\dagger$ ).

It is observed that all the elements are uniformly distributed throughout Ce-CHT/PVA composite sample. The EDAX of CeCHT/PVA composite nanofiber [Fig. 3(a)] show the presence of $\mathrm{C}, \mathrm{O}$ and Ce only. However, Ce-CHT/PVA composite nanofibers, after the adsorption of As(III), indicate the presence of As along with $\mathrm{C}, \mathrm{O}$ and $\mathrm{Ce}$ [Fig. 3(b)]. A comparison of Fig. 3(a) and (b) also reveals the change in the morphology of Ce-CHT/PVA composite nanofibers after As(III) adsorption.

\subsection{Effect of $\mathrm{pH}$, Concentration of $\mathrm{Ce}(\mathrm{III})$ and temperature on adsorption of As(III)}

The $\mathrm{pH}$ and $\mathrm{pH}_{\mathrm{pzc}}$ of a solution have a significant effect on adsorption at the liquid-solid interface. Arsenic can exist in several oxidation states, such as $-3,0,+3$ and +5 , however, in natural water, it exists in inorganic form as the oxyanions of trivalent arsenite or pentavalent arsenate. The effect of $\mathrm{pH}$ on the \% removal of As(III) has been investigated by varying $\mathrm{pH}$ from 2 to 12. As shown in Fig. 1(b), on increasing the $\mathrm{pH}$ of the test solution from 2 to 6.2, the removal of As(III) increases continuously, while on increasing $\mathrm{pH}$ above 7.0, the adsorption of As(III) decreases gradually. The point zero charge of Ce-CHT/ PVA composite nanofiber is found to be 7.34 [Fig. 1(a)]. At $\mathrm{pH}$ less than $\mathrm{pH}_{\mathrm{PZC}}$ (7.34), the surface of adsorbent would considered to be positively charged and would be involved in the protonation of $-\mathrm{NH}_{2}$ group, resulting in attracting the oxyanions of As. However, at $\mathrm{pH}$ above $\mathrm{pH}_{\mathrm{PZC}}$, the surface of adsorbent is considered to be negatively charged and attract cations. As shown in Fig. 1(b), the maximum adsorption was observed in $\mathrm{pH}$ range of 6.2-7.0. At this $\mathrm{pH}$ range (6.2-7.0), $-\mathrm{NH}_{2}$ groups of Ce-CHT/PVA nanofibers are positively charged and are involved in the protonation of amino group $\left(-\mathrm{NH}_{2}\right.$ to $\mathrm{NH}_{3}{ }^{+}$), which leads to the interaction of As(III), while at basic $\mathrm{pH}$, protonation does not occur and as a result the adsorption decreases. The $\mathrm{pH}$ of the test solution is tested before and after the adsorption of $\mathrm{As}(\mathrm{III})$ and no significant change in $\mathrm{pH}$ is observed. Therefore, it can be inferred that Ce-CHT/PVA composite nanofiber is insoluble in water.

The effect of various concentration of Ce(III) nitrate hexahydrate $(0.5-5 \%)$ in CHT/PVA composite nanofibers for As(III) removal is investigated at ambient temperature. As shown in Fig. 4, the adsorption of As(III) increases on increasing the concentration of $\mathrm{Ce}(0.5$ to $3.5 \%)$ in $\mathrm{CHT} / \mathrm{PVA}$ composite nanofibers. This happens because on increasing the concentration of $\mathrm{Ce}(\mathrm{III})$, the positive charge on chitosan increases. Above 3.5 weight $\%$ of $\mathrm{Ce}(\mathrm{III})$ in $\mathrm{CHT} / \mathrm{PVA}$, the viscosity of the solution is increased, resulting in the formation of beaded and non uniform Ce-CHT/PVA composite nanofibers and decreased adsorption of $\operatorname{As}(\mathrm{III})$.
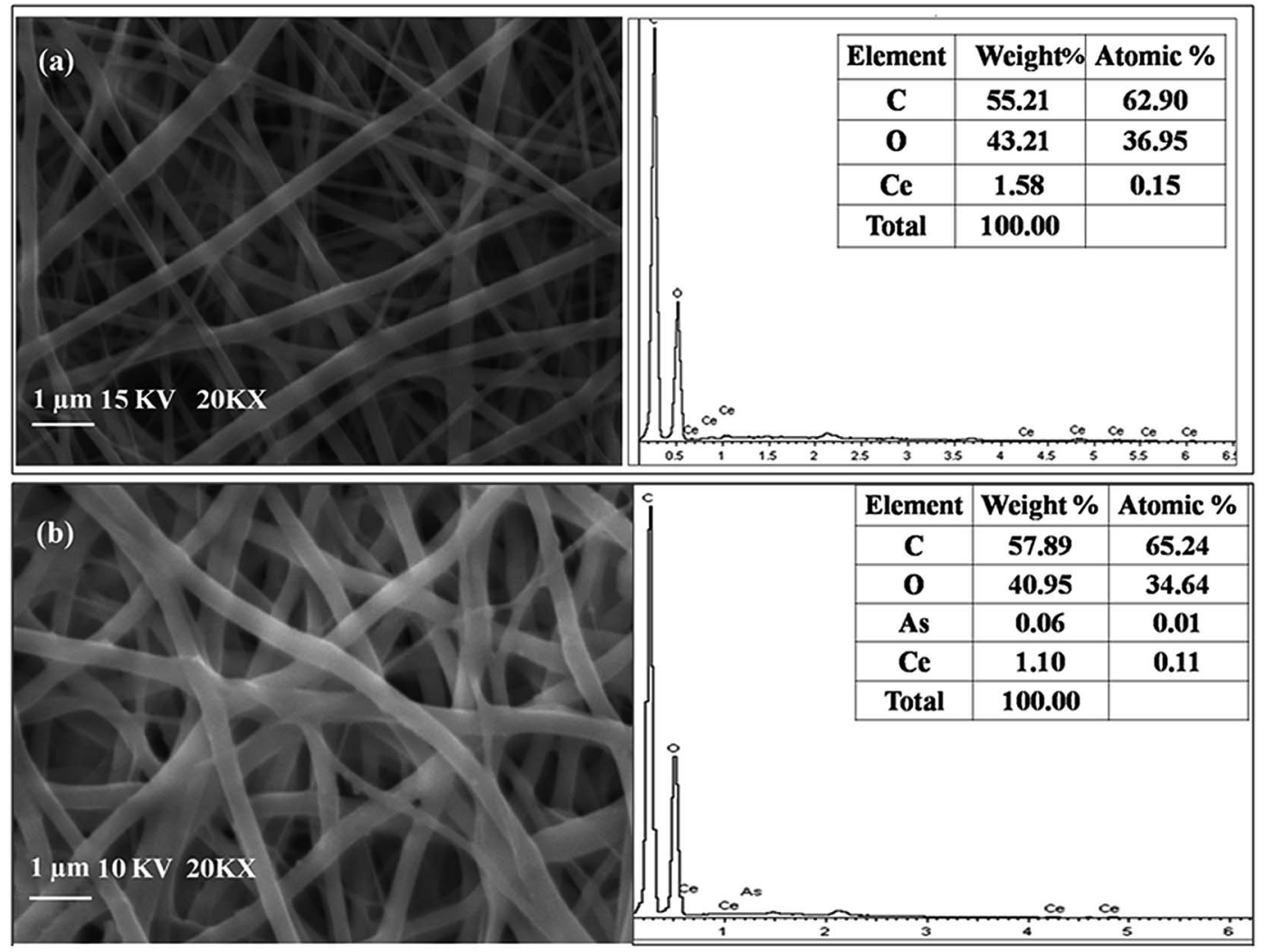

Fig. 3 SEM and EDAX of Ce-CHT/PVA composite nanofibers (a) before and (b) after adsorption. 


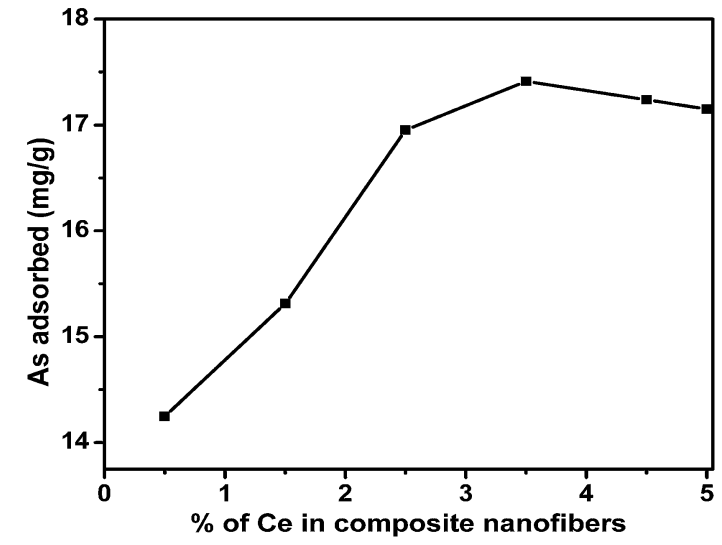

Fig. 4 Effect of \%Ce As(III) adsorption.

The effect of temperature on As(III) adsorption is investigated by varying temperature $\left(10-65^{\circ} \mathrm{C}\right)$ at optimized $\mathrm{pH}$. As shown in ESI Fig. S2, $\dagger$ the maximum adsorption is observed between 20 and $35{ }^{\circ} \mathrm{C}$. Increase in temperature $\left(>35^{\circ} \mathrm{C}\right)$ or decrease $\left(<20^{\circ} \mathrm{C}\right)$ result in the decrease of $\mathrm{As}(\mathrm{III})$ adsorption. This could be attributed to the protonation of $-\mathrm{NH}_{2}$ groups, which remain unaffected probably due to the surface complexes and interactions. $^{40}$

Fig. S3† shows the FTIR spectra of CHT/PVA, Ce-CHT/PVA and As(III) adsorbed Ce-CHT/PVA composite nanofibers. The shifting of adsorption peaks at $1363 \mathrm{~cm}^{-1}$ and $1075 \mathrm{~cm}^{-1}$ [Fig. S3(a)†] toward higher wave numbers at $1371 \mathrm{~cm}^{-1}$ and $1095 \mathrm{~cm}^{-1}$ [Fig S3(b)†] may be due to the interaction of $-\mathrm{NH}$ group with cerium(III) in Ce-CHT/PVA. ${ }^{30}$ After the adsorption of As(III) (curve 'c', Fig. S3†), it is observed that the peak intensity increases due to the interaction of As(III) with $-\mathrm{NH}_{2}\left(1643 \mathrm{~cm}^{-1}\right.$, $\left.1579 \mathrm{~cm}^{-1}\right)$ and $\mathrm{Ce}\left(1376 \mathrm{~cm}^{-1}, 1095 \mathrm{~cm}^{-1}\right) .{ }^{40-42}$ From the figure, it is deduced that the peak positions shifts towards higher wave number after the adsorption of As(III) on the Ce-CHT/PVA composite nanofibers surface.

In XRD spectra (ESI Fig. S4 $\dagger$ ) of Ce-CHT/PVA shifting of absorption peaks from $2 \theta=9.16^{\circ}$ and $19.32^{\circ}$ (ref. 43 and 44 ) in (Fig. S4, curve a $\dagger$ ) to $2 \theta=9.3^{\circ}$ and $19.86^{\circ}$ (Fig. S4, curve ' $\mathrm{b} \nmid \dagger$ ), confirming the As(III) adsorption on Ce-CHT/PVA composite nanofibers. The literature reveals that peaks at $34^{\circ}$ and $37^{\circ}$ are due the ceria structure. ${ }^{45}$ The peaks at $38^{\circ}$ and $45^{\circ}$ may have appeared due the introduction of cerium in CHT/PVA matrix, resulting in the formation of unknown crystallite phases. The results are in agreement with the observations of SEM and FTIR.

On the basis of SEM-EDAX, FTIR and XRD studies, it is evident that As(III) interacts with Ce-CHT/PVA on - NH and Ce(III) sites. The electrospun composites nanofibers of CHT, PVA and cerium(III) may be considered as the formations of complex of chitosan with PVA and Ce(III). In acidic solutions, more positively charged surface sites are developed by the protonation of amino groups on chitosan and highly electropositive Ce(III), which efficiently attract As(III). A graphical representation of the Ce-CHT/PVA composite nanofibers interacting with As(III) is given in Fig. 5. The adsorption studies are carried out in the $\mathrm{pH}$ range of 6.2-7.0. CSIR-NPL certified reference material was used

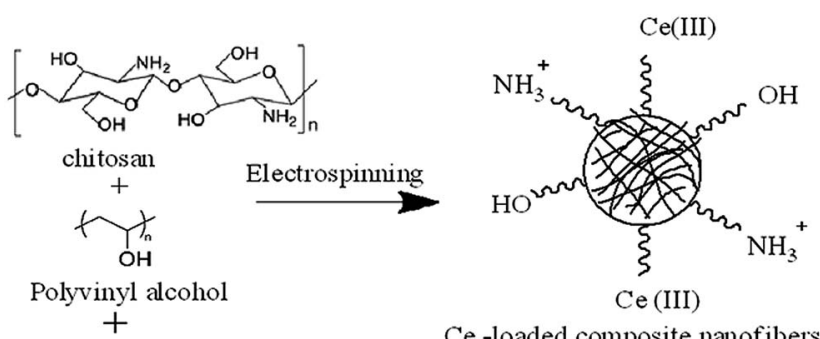

Ce (III)

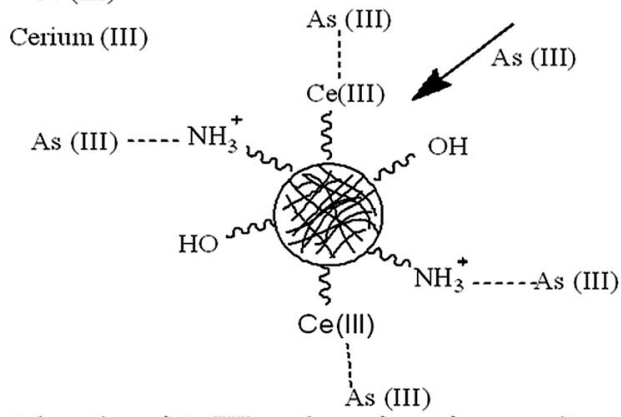

Adsorption of As(III) on the surface of composite nanofibers

Fig. 5 Probable reaction mechanism of As(III) adsorption on Ce-CHT/ PVA composite nanofibers.

for As removal studies. The CRM was prepared in nitric acid after dissolving high purity arsenic oxide powder. NIST (USA) certified reference material was used for the calibration of equipment and was prepared in nitric acid. $\mathrm{H}_{3} \mathrm{AsO}_{3}$ is a neutral species $(\mathrm{pH}<9.2)$, and within 6.2-7.0 $\mathrm{pH}$ range, As(III) interacts with $-\mathrm{NH}_{2}$, and $\mathrm{Ce}$ (III) groups attach to Ce-CHT/PVA and adsorb over the surface of composite nanofibers. ${ }^{46}$ This is also evident from FTIR results (ESI Fig. S3 $\dagger$ ). The adsorption peaks at 1643 $\mathrm{cm}^{-1}$ and $1579 \mathrm{~cm}^{-1}$ in curve 'b' shifted to $1656 \mathrm{~cm}^{-1}$ in curve 'c', confirming the adsorption of As(III) on the surface of CeCHT/PVA composite nanofibers. The increased intensity of FTIR peaks for adsorbed Ce-CHT/PVA nanofibers (ESI Fig. S3 $\dagger$ ) is also an indication of the adsorption of As(III) on composite surface.

\subsection{Kinetic study}

The kinetic study of the adsorption of As(III) on Ce-CHT/PVA composite nanofiber is carried out to evaluate the equilibrium time required in this process and the rate of adsorption of As(III) on Ce-CHT/PVA composite nanofibers. The experiments are carried out with $3.5 \mathrm{mg} \mathrm{L}{ }^{-1}$ concentration of As(III) in optimum conditions. The results of kinetic studies are given in Fig. 6. It is observed from the figure that the adsorption of As(III) gradually increases and more than 98\% adsorption occurs within 60 minutes. However, the removal rate of As(III) was higher in the first ten minutes probably due to the availability of more free sites in Ce-CHT/PVA composite nanofibers. Adsorption rates gradually decrease because of an increased occupancy of free sites by As(III). The maximum adsorption occurs within the first 60 minutes and after that the adsorption of As(III) becomes stagnant. Fig. 6(a) represents the effect of time on adsorption capacity (' $t$ ' $v s$. ' $q_{t}^{\prime}$ '), whereas Fig. 6 (b) shows the regression curve $\left(t / q_{t} v s\right.$. ' $t$ '). The adsorption capacity $\left(q_{\mathrm{e}}\right)$, initial adsorption rate 

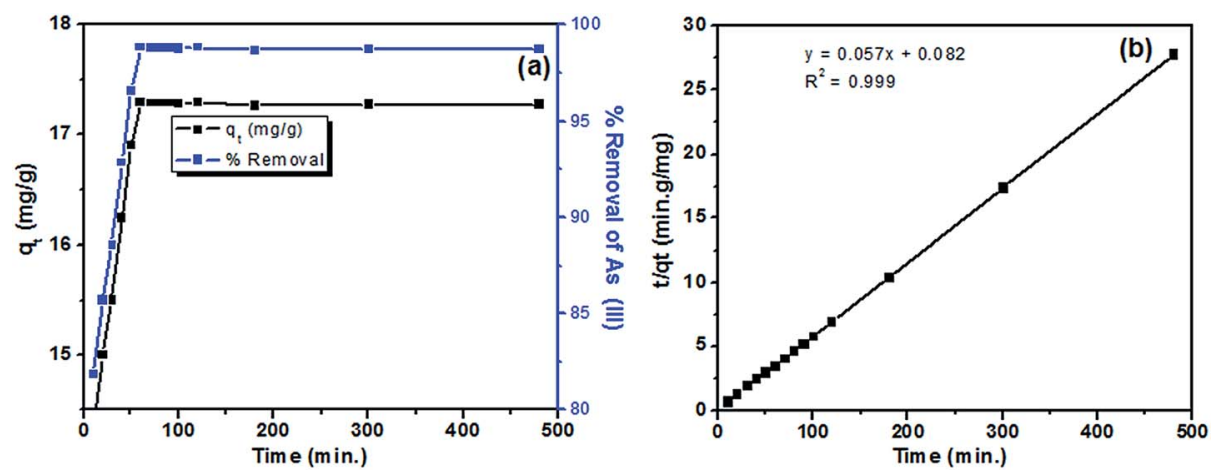

Fig. 6 (a) Effect of time on the adsorption capacity of As(III), (b) pseudo second order kinetics.

$(h)$, rate constant $(k)$ and coefficient of determination $\left(R^{2}\right)$ are obtained from the linear graph between ' $t / q_{t}$ ' and time. Pseudo second order kinetic equation used is given as:

$$
\frac{t}{q_{t}}=\frac{1}{h}+\frac{t}{q_{t}}
$$

when ' $t$ ' is equal to zero initial adsorption rate, ' $h$ ' may be calculated as:

$$
h=k q_{\mathrm{e}}^{2}
$$

where ' $q_{\mathrm{e}}$ ' and ' $q_{t}$ ' are the adsorption capacity of As(III) in 'mg $\mathrm{g}^{-1}$ ' at equilibrium and at time ' $t$ ' respectively. ' $h$ ' represents the initial adsorption rate $\left(\mathrm{mg} \mathrm{g}^{-x} \mathrm{~min}^{-1}\right)$ and ' $k$ ' represents the rate constant $\left(\mathrm{g} \mathrm{mg}^{-x} \mathrm{~min}^{-1}\right)$. The adsorption capacity ' $q_{\mathrm{e}}$ ' of the prepared Ce-CHT/PVA composite nanofibers was determined, which was $c a .17 .54\left(\mathrm{mg} \mathrm{g}^{-1}\right)$. From the regression graph, the value of ' $h$ ', ' $k$ ' and $R^{2}$ are found to be $12.19 \mathrm{mg} \mathrm{g}^{-1} \mathrm{~min}^{-1}, 0.04 \mathrm{~g}$ $\mathrm{mg}^{-1} \mathrm{~min}^{-1}$ and 0.999 , respectively. It can be concluded from the results that the adsorption process of As(III) by Ce-CHT/PVA composite nanofibers fits the pseudo-second order kinetic model.

\subsection{Adsorption isotherm model}

There are different adsorption isotherm models for understanding solid-liquid interface such as Langmuir, Freundlich, Temkin and Dubinin-Radushkevich (D-R) isotherm. Among these, Langmuir and Freundlich are the most commonly used models for verifying the relationship between solid-liquid interfaces. ${ }^{47}$ The equilibrium studies are conducted for $10 \mathrm{mg}$ of Ce-CHT/PVA composite nanofibers in $50 \mathrm{~mL}$ solutions, where the concentration of $\operatorname{As}(\mathrm{III})$ is varied from 100 to $4500 \mu \mathrm{g}$. After 60 minutes, the remaining concentrations of $\mathrm{As}(\mathrm{III})$ ' $C_{\mathrm{f}}$ ' were measured. Fig. 7(a) indicates that the As(III) adsorption capacity of Ce-CHT/PVA increases gradually with increasing concentrations of As(III). It is further observed that $10 \mathrm{mg}$ of Ce-CHT/PVA removes up to $1500 \mu \mathrm{g} \mathrm{L}^{-1}$ concentration of $\mathrm{As}(\mathrm{III})$ and the maximum adsorption capacity ' $q_{\mathrm{m}}$ ' is observed at $3500 \mu \mathrm{g} \mathrm{L} \mathrm{L}^{-1}$ concentration of $\mathrm{As}(\mathrm{III})$. No further adsorption occurs on increasing the concentration of metal ion due to the saturation of active sites in Ce-CHT/PVA composite nanofibers. The findings of the present study were applied to Langmuir isotherm model, which is based on the assumption that interactive force between the adsorbed molecules is negligible and once the active site is filled, no more adsorption occurs. The Langmuir isotherm equation ${ }^{27,48}$ used for calculation is expressed as:

$$
\frac{C_{\mathrm{f}}}{q_{\mathrm{e}}}=\frac{1}{K_{\mathrm{L}} q_{\mathrm{m}}}+\frac{C_{\mathrm{f}}}{q_{\mathrm{m}}}
$$

where ' $\mathrm{q}_{\mathrm{e}}$ ' $\left(\mathrm{mg} \mathrm{g}^{-1}\right)$ is the amount of As(III) adsorbed on Ce-CHT/ PVA composite nanofibers, ' $C_{\mathrm{f}}$ ' $\left(\mu \mathrm{g} \mathrm{L}^{-1}\right)$ is the final concentration of As(III) remaining in the test solution, ' $q_{\mathrm{m}}$ ' is the maximum adsorption capacity in $\mathrm{mg} \mathrm{g}^{-1}$, ' $q_{\mathrm{e}}$ ' $\left(\mathrm{mg} \mathrm{g}^{-1}\right)$ is the adsorption capacity at equilibrium, and $K_{\mathrm{L}}\left(\mathrm{L} \mathrm{mg}^{-1}\right)$ is the energy of reaction. The value of ' $q_{\mathrm{m}}$ ' (maximum adsorption capacity) and ' $K_{\mathrm{L}}$ ' (energy of reaction) can be determined by the slope and intercept of a linear plot of $C_{\mathrm{f}} / q_{\mathrm{e}}$ against ' ${ }^{\mathrm{f}}$ ', as shown in Fig. 7(b). The maximum adsorption capacity of the composite using Langmuir isotherm is found to be $18.0 \mathrm{mg} \mathrm{g}^{-1}$, whereas ' $K_{\mathrm{L}}$ ' and ' $R^{2}$ ' are observed to be $0.12 \mathrm{~L} \mathrm{mg}^{-1}$ and 0.999 , respectively. The experimental data fitted to Langmuir isotherm suggests a monolayer adsorption of As(III) on the surface of CeCHT/PVA composite nanofibers. The adsorption behavior of CHT/PVA composite nanofibers is also examined by varying the initial concentration of As(III) (100-4500 $\left.\mu \mathrm{g} \mathrm{L}^{-1}\right)$ at optimized conditions. The observed efficiency was only 30-35\%. Effect of initial concentration on $\mathrm{As}$ (III) adsorption and Langmuir isotherm demonstrate that the adsorption capacity of CHT/PVA composite nanofibers is quite low (ESI Fig. S6†).

The separation factor ' $R_{\mathrm{L}}$ ' is an important feature of Langmuir isotherm to test the feasibility of process, which was calculated using eqn (6).

$$
R_{\mathrm{L}}=\frac{1}{1+1\left(K_{\mathrm{L}} C_{\mathrm{i}}\right)}
$$

where $C_{0}=$ initial concentration and $K_{\mathrm{L}}=$ Langmuir constant; energy of adsorption. $R_{\mathrm{L}}$ value indicates whether adsorption nature is unfavorable $(>1)$, linear $(=1)$, favorable $(>0$ or $<1)$ and irreversible $(=0)$. In the present study, the value of $R_{\mathrm{L}}$ calculated from eqn (6) is found to be 0.90 ( $>0$ or $<1$ ), suggesting favorable adsorption of $\mathrm{As}(\mathrm{III})$ on the surface of Ce-CHT/PVA composite nanofibers.

Quality assurance is an important aspect for the determination of toxic species such as arsenic. The quality and 

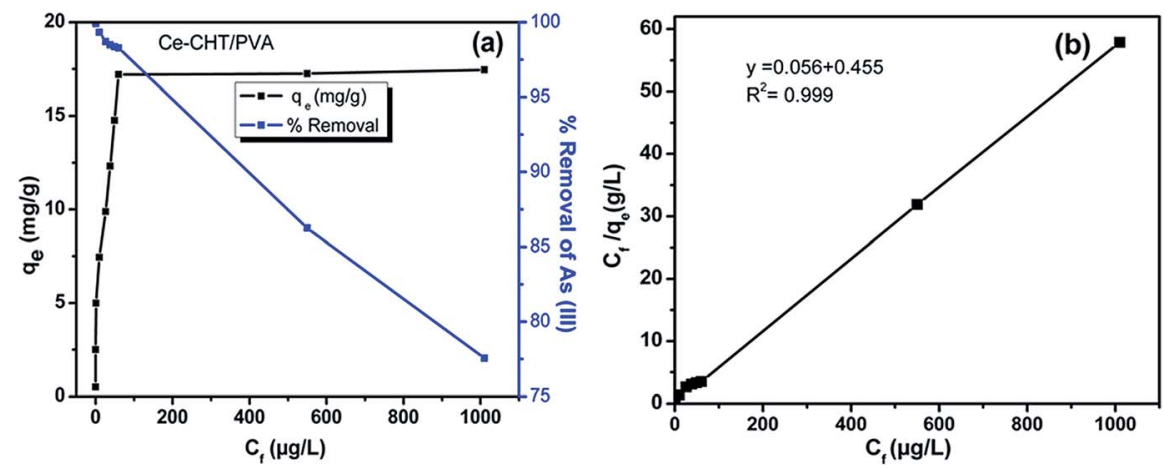

Fig. 7 (a) Effect of initial concentration of As(III) on adsorption and (b) Langmuir adsorption isotherm of Ce-CHT/PVA composite nanofibers.

reliability of data depends upon uncertainty measurements at each step involved during measurement. There are many steps in the quantification of As(III) by AAS-HG such as repeatability, weighing, reference material, volumetric flask, pipettes, etc. The calibration of AAS-HG was done using NIST-SRM (9.999 \pm 0.015 $\mathrm{mg}^{-1}$ ) following appropriate dilutions in the range of analyte sample. A process blank was also employed and corrections were applied wherever required. Mean concentration values of triplicate for each sample were considered for the calculation of As(III) concentration. The major sources of uncertainty have been included in the combined uncertainty according to EURACHEM/GUM guidelines. ${ }^{\mathbf{4 9 , 5 0}}$ For example the measurement uncertainty for $1000 \mu \mathrm{g} \mathrm{L}^{-1} \mathrm{As}(\mathrm{III})$ standard solution has been carried out and potential sources (as given in Table 1) are considered for combined uncertainty. On the basis of measurement, the results for $1000 \mu \mathrm{g} \mathrm{L}^{-1}$ standard solution are found to be $999.6 \pm 5.4\left(\mu \mathrm{g} \mathrm{L}^{-1}\right)$ with $95 \%$ confidence level $(K=2)$, as given in Table 1 . In the same manner, various concentration of As(III) are considered and same parameters are considered for final calculation.

The proposed material (Ce-CHT/PVA composite nanofiber) are compared with other chitosan based adsorbents cited in the literature (Table 2). It could be seen from the table that the proposed material have several advantages in terms of speed and efficiency of As(III) adsorption in comparison to those cited in literature. ${ }^{\mathbf{1 4 2 4 - 2 9}}$

\subsection{Interference studies}

The performance of Ce-CHT/PVA composite nanofibers $(10 \mathrm{mg})$ for $\mathrm{As}(\mathrm{III})$ removal is carried out individually for $\mathrm{Mg}, \mathrm{Ca}, \mathrm{Pb}, \mathrm{Zn}$, $\mathrm{Cu}, \mathrm{Fe}, \mathrm{NO}_{3}{ }^{-}, \mathrm{Cl}^{-}$and $\mathrm{SO}_{4}{ }^{-}$ions. Equimolar quantity of each ion with As(III) were taken. The assessment was carried out with $50 \mathrm{~mL}$ analyte solution at $6.2-7.0 \mathrm{pH}$ range. The results are presented in Table 3.

It is observed from the table that $\mathrm{Pb}, \mathrm{NO}_{3}{ }^{-}$, and $\mathrm{SO}_{4}{ }^{-}$ compete with As(III) during adsorption, whereas $\mathrm{Ca}, \mathrm{Zn}, \mathrm{Mg}, \mathrm{Cu}$ and $\mathrm{Cl}^{-}$show less interference. However, the composite fibres show higher selectivity towards As(III). Further, As(III) removal experiment was also carried out for As(III) solution having multi elements (total $800 \mu \mathrm{g} \mathrm{L} \mathrm{L}^{-1}$ or $100 \mu \mathrm{g} \mathrm{\textrm {L } ^ { - 1 }}$ each) at optimized conditions. In this experiment, the As(III) adsorption on Ce-PVA/ CHT composite nanofibers decreases by $25-35 \%$, indicating interference by other ions.

\subsection{Recycling of adsorbent}

To check the reusability of Ce-CHT/PVA composite nanofibers, the test material was regenerated and used twice. Regeneration involved treating the fiber with $0.01 \mathrm{M} \mathrm{HCl}$ followed by thorough rinsing with de-ionized water. Initially, the prepared composite nanofiber removes As(III) completely. However, in second and third cycle, the As removal dropped to $65 \%, 35 \%$, respectively. The reduction in adsorption could be attributed to the deformation of nanofibers in $\mathrm{HCl}$ medium during regeneration.

The quality of water before the addition of adsorbent and after the removal of As(III) is assessed by obtaining UV visible spectra (ESI Fig. S5(a) \& (b) †). No peak was observed at 272 $\mathrm{nm}$ after the removal of As(III). UV-vis spectra thus conclude that a Ce-CHT/PVA composite nanofiber removes As(III) efficiently. This information further supports the findings of AAS-HG.

Table 1 Major sources of uncertainty in the measurement of As(III) by AAS-HG

\begin{tabular}{|c|c|c|c|c|c|c|}
\hline Parameters & Value; $x$ & Unit & Standard uncertainty; $u(x)$ & Unit & $u(x) / x$ & $U_{\mathrm{c}}(K=2)$ \\
\hline Repeatability & 999.6 & $\mu g \mathrm{~L}^{-1}$ & 0.310 & $\mu g \mathrm{~L}^{-1}$ & 0.00031 & 2.7 \\
\hline NIST SRM, 3103a & 9.999 & $\mathrm{mg} \mathrm{g}^{-1}$ & 0.015 & $\operatorname{mg} g^{-1}$ & 0.0015 & \\
\hline Volumetric flask & 50 & $\mathrm{~mL}$ & 0.01 & $\mathrm{~mL}$ & 0.00020 & \\
\hline Weighing balance & 0.1 & $\mathrm{~g}$ & 0.0001 & $\mathrm{~g}$ & 0.00100 & \\
\hline Pipette; suction & 5.0 & $\mathrm{~mL}$ & 0.01 & $\mathrm{~mL}$ & 0.00200 & \\
\hline
\end{tabular}


Table 2 Adsorption comparison of Ce-CHT/PVA composite nanofibers and cited adsorbent

\begin{tabular}{lcll}
\hline Name of adsorbent & $\begin{array}{l}\text { Adsorption capacity } \\
\left(\mathrm{mg} \mathrm{g}^{-1}\right)\end{array}$ & Time & pH/Temp. \\
\hline Iron-impregnated chitosan & 6.48 & $2 \mathrm{~h}$ & $8 / 25$ \\
Chitosan beads & 1.83 & $24 \mathrm{~h}$ & $5 / 25$ \\
Porous $\mathrm{CS} / \mathrm{Fe}_{3} \mathrm{O}_{4} / \mathrm{Fe}(\mathrm{OH})_{3}$ microsphere & 8.47 & $45 \mathrm{~min}$ & No information \\
Iron-chitosan composite granules & 16.15 & $4 \mathrm{~h}$ & $7 / 25$ \\
Alpha-Fe $\mathrm{O}_{3}$, impregnated chitosan beads & 6.18 & $6 \mathrm{~h}$ & $5 / 30$ \\
Chitosan-Fe-crosslinked complex & 13.4 & 60 min & $9 / 25$ \\
Cerium-loaded cation exchange resin & 2.59 & No information & $5-6$ \\
Ce-CHT/PVA composite nanofibers & 18.2 & 60 min & $6.2-7.0 /\left(20-35{ }^{\circ} \mathrm{C}\right)$
\end{tabular}

Table 3 Removal of As(III) in presence of individual ions

\begin{tabular}{|c|c|c|c|}
\hline As with $\mathrm{Pb}$ & 1000 & 1000 & 28.3 \\
\hline As with $\mathrm{Mg}$ & 1000 & 1000 & 1.4 \\
\hline As with $\mathrm{Ca}$ & 1000 & 1000 & 3.6 \\
\hline As with $\mathrm{Cl}^{-}$ & 1000 & 1000 & 11.2 \\
\hline As with $\mathrm{NO}_{3}{ }^{-}$ & 1000 & 1000 & 23.8 \\
\hline As with $\mathrm{SO}_{4}^{-}$ & 1000 & 1000 & 38.4 \\
\hline
\end{tabular}

\section{Conclusions}

In the present investigation, Ce-CHT/PVA composite nanofibers prepared through electrospinning technique showed efficient removal of As(III) from water. The proposed material has a high surface area and more active sites for As(III) adsorption than other adsorbents reported in literature. SEM-EDAX, FTIR and XRD also confirm As(III) adsorption over the surfaces of Ce-CHT/ PVA composite nanofibers. The kinetic study of adsorption reveals that over $80 \%$ of As(III) can be removed within the first 10 minutes. The experimental data follows Langmuir isotherm model with adsorption capacity of $18.0 \mathrm{mg} \mathrm{g}^{-1}$. The value appears to be better than several other chitosan based adsorbents. The quality of water was further confirmed by UV-vis spectroscopy before and after As(III) removal. The effects of several common ions present in water $(\mathrm{Mg}, \mathrm{Ca}, \mathrm{Pb}, \mathrm{Zn}, \mathrm{Cu}$, $\mathrm{SO}_{4}{ }^{-}, \mathrm{NO}_{3}{ }^{-}, \mathrm{Cl}^{-}$) on the adsorption of As(III) were tested individually and with multi-element solution. The results show favorable selectivity for As(III). Therefore, it may be concluded that the proposed material can be used efficiently for arsenic removal in the presence of several tested ionic species. The literature further reveals that the majority of adsorption processes required one oxidizing and one adsorbing agent for the removal of toxic elements, while the material under study adsorbs As(III) efficiently without the presence of an oxidizing agent. Therefore, water can be safely used for potability as well as other applications. The prepared material (Ce-CHT/PVA) purifies water below the prescribed limit of WHO/EPA up to $1500 \mu \mathrm{g} \mathrm{L}^{-1}$. In addition, Ce-CHT/PVA composite nanofibers are nontoxic and can be used for water purification as such or after embedding them on non-woven fabrics, porous membranes or candles. Because of rapid and high adsorption of As(III), such materials can be useful in areas with no access to electricity.

\section{Acknowledgements}

The authors are grateful to Director, CSIR-National Physical Laboratory, New Delhi for encouragement and permission to publish this work. The authors are also grateful to Mr Jai Tawale for providing SEM analysis and Dr S. Swarupa Tripathy for their valuable suggestions.

\section{References}

1 Y. Zhang, M. Yang and X. Huang, Chemosphere, 2003, 51, 945-952.

2 L. H. Keith and W. A. Telliard, Environ. Sci. Technol., 1979, 13, 416-424.

3 R. Hübner, K. B. Astin and R. J. H. Herbert, J. Environ. Monit., 2010, 12, 1511-1514.

4 P. Singh, J. Bajpai, A. K. Bajpai and R. B. Srivatava, J. Chin. Chem. Soc., 2008, 55, 952-961.

5 R. Rakhunde, D. Jasudkar, L. Deshpande, H. D. Juneja and P. K. Labhasetwar, International Journal of Environmental Sciences and Research, 2012, 1, 92-96.

6 M. Burguera and J. L. Burguera, Talanta, 1997, 44, 15811604. 
7 S. Yamamura, Drinking Water Guidelines and Standards, World Health Organization, Geneva, Switzerland, 2001.

8 Y. Lee, I. H. Um and J. Yoon, J. Environ. Sci. Technol., 2003, 37, 5750-5756.

9 S. Bang, G. P. Korfiatis and G. P. Meng, J. Hazard. Mater., 2005, 121(1-3), 61-67.

10 Technologies and Costs for Removal of Arsenic from Drinking Water, EPA 815-R-00 028, December 2000, http:// www.epa.gov/safewater.

11 L. C. Roberts, S. J. Hug, T. Ruettimann, M. Billah, A. W. Khan and M. T. Rahman, Environ. Sci. Technol., 2004, 38, 307-315.

12 X. Leupin and S. J. Hug, Water Res., 2005, 39, 1729-1740.

13 D. D. Gang, B. Deng and L. S. Lin, J. Hazard. Mater., 2010, 182, 156-161.

14 A. K. Gupta, D. Deva, A. Sharma and N. Verma, Ind. Eng. Chem. Res., 2010, 49, 7074-7084.

15 M. Salim and Y. Munekage, Int. J. Environ. Res., 2009, 3, 1322.

16 Z. Li, S. Deng, G. Yu and J. Huang, Chem. Eng. J., 2010, 161, 106-113.

17 W. Bao, W. Haobin, Y. Le, X. Rong, T. T. Lim and L. W. Xiong (David), Adv. Mater., 2012, 24, 111-1116.

18 Y. X. Bao, T. Yan, X. Wang and L. W. Xiong (David), Mater. Horiz., 2014, 1, 379-399.

19 Z. Elwakeel, Desalination, 2010, 250, 105-112.

20 S. Roller and N. Covill, Int. J. Food Microbiol., 1999, 47, 67-77.

21 H. Yao, L. Guo, B. H. Jiang, J. Luo and X. Shi, J. Environ. Pathol., Toxicol. Oncol., 2008, 27, 77-88.

22 Q. Li, E. T. Dunn, E. W. Grandmaisson and M. F. A. Goosen, Applications and Properties of Chitosan, in Applications of Chitin and Chitosan, ed. M. F. A. Goosen, Technomic Publishing, Lancaster, USA, 1997, pp. 3-29.

23 V. M. Srinivasan, M. Mishra, J. S. Paliwal, S. K. Singh, E. Selvarajan, V. Suganthi and C. S. Dev, Biotechnology, 2014, 4, 167-175.

24 C. C. Chen and Y. C. Chung, J. Environ. Sci. Health, Part A: Toxic/Hazard. Subst. Environ. Eng., 2006, 41, 645-658.

25 A. Gupta, V. S. Chauhan and N. S. Krishnan, Water Res., 2009, 43, 3862-3870.

26 D. T. Vu, X. Li and C. Wang, Sci. China: Chem., 2013, 5(6), 678-684.

27 B. Liu, D. Wang, H. Li, Y. Xu and L. Zhan, Desalination, 2011, 272, 286-292.

28 H. H. D Santos, C. A. Demarchi, C. A. Rodrigues, J. M. Greneche, N. Nedelko and A. Ś. Waniewska, Chemosphere, 2011, 82, 278-283.

29 Z. He, S. Tian and P. Ping, J. Rare Earths, 2012, 30, 563-572.
30 F. Wang and M. Ge, Text. Res. J., 2012, 1-10, DOI: 10.1177/ 0040517512454188.

31 Y. G. Li, Z. R. Yang and J. Cheng, J. Rare Earths, 2007, 25, 452456.

32 A. Sharma, A. Gupta, G. Rath, A. Goyal, R. B. Mathur and S. R. Dhakate, J. Mater. Chem. B, 2013, 1, 3410-3418.

33 S. Tripathy, G. K. Mehrotra and P. K. Dutta, Int. J. Biol. Macromol., 2009, 45, 372-376.

34 S. R. Dhakate, B. Singla, M. Uppal and R. B. Mathur, Adv. Mater. Lett., 2011, 1, 200-204.

35 S. R. Dhakate, A. Gupta, A. Chaudhari, J. Tawale and R. B. Mathur, Synth. Met., 2011, 161, 411-419.

36 A. Gupta, S. Dhakate, M. Pahwa, S. Sinha, S. Chand and R. B. Mathur, Process Biochem., 2013, 48, 124-132.

37 N. Fiol and I. Villaescusa, Environ. Chem. Lett., 2009, 7, 7984.

38 N. Singh, A. Sarkar, R. Ramchandran and K. Lal, Asian J. Chem., 2003, 15, 1327-1330.

39 B. M. Min, S. W. Lee, J. N. Lim, Y. You, T. S. Lee, P. H. Kang and W. H. Park, Polymer, 2004, 45, 7137-7142.

40 A. Tiwari, T. Dewangana and A. K. Bajpai, J. Chin. Chem. Soc., 2008, 55, 952-961.

41 U. K. Parida, A. K. Nayak, B. K. Binhani and P. L. Nayak, J. Biomater. Nanobiotechnol., 2011, 2, 414-425.

42 N. Singh, Rashmi, S. Singh, D. Soni, R. Pasricha, P. K. Gupta, A process for the synthesis of $\mathrm{ZnO}_{2}$ nanomaterial, USA, FP05990/DKT (Continuation of FP04772), FP04773/PC (South Africa).

43 K. Nakane, T. Yamashita, K. Iwakura and F. Suzuki, J. Appl. Polym. Sci., 1999, 74, 133-138.

44 R. J. Samuels, J. Polym. Sci., Polym. Phys. Ed., 1981, 19, 10811105.

45 V. B. Mane, L. H. Mahind, K. D. Jadhav, S. A. Waghmode and S. P. Dagade, Carbon: Sci. Technol., 2013, 260-264.

46 N. Singh, S. P. Singh, V. Gupta, H. K. Yadav, T. Ahuja, S. S. Tripathy and Rashmi, Environ. Prog. Sustainable Energy, 2013, 32, 1023-1029.

47 A. O. Dada, A. P. Olalekan, A. M. Olatunya and O. DADA, J. Appl. Chem., 2012, 3, 38-45.

48 B. Chen, Z. Zhu, J. Ma, Y. Qiua and J. Chen, J. Mater. Chem. A, 2013, 1, 11355.

49 European Standard EN ISO/IEC 17025, General Requirements for the Competence of the Testing laboratories, European Committee for Standardization, Brussels, 2000, p. 14.

50 ISO (1995) Guide (GUM) 2nd Edition, International Organization for Standardization, to Expression of Uncertainty in Measurement (Geneva), Switzerland. 